\title{
The protective effect of lycopene supplement against vancomycin-induced nephrotoxicity; a randomized double-blind placebo-controlled clinical trial
}

\author{
Somayeh Haghighipour ${ }^{1}$, Rasool Soltani², Ali Anjomshoa ${ }^{3 *}$ \\ ${ }^{1}$ Infectious Disease and Tropical Medicine Research Center, Isfahan University of Medical Sciences, Isfahan, Iran \\ ${ }^{2}$ Department of Clinical Pharmacy and Pharmacy Practice, Faculty of Pharmacy, Isfahan University of Medical Sciences, Isfahan, Iran \\ ${ }^{3}$ Department of Infectious Diseases, School of Medicine, Isfahan University of Medical Sciences, Isfahan, Iran
}

\section{A R T I C L E IN F O}

Article Type:

Original

\section{Article History:}

Received: 4 May 2020

Accepted: 17 July 2020

Published online: 28 July 2020

Keywords:

Vancomycin

Nephrotoxicity

Lycopene

\begin{abstract}
A B S T R A C T
Introduction: It has been proposed that oxidative stress plays a crucial role in vancomycininduced nephrotoxicity (VIN).

Objectives: The present study aimed to investigate the nephroprotective effects of lycopene, as a powerful antioxidant, on VIN.

Patients and Methods: In the present study, individuals who received vancomycin (VCM) for any indication were assigned to drug $(n=28)$ and control $(n=30)$ groups. The individuals in the drug group received $25 \mathrm{mg}$ of oral lycopene daily for 10 days started concurrently with VCM and the patients in the placebo group received placebo tablets with VCM. Serum levels of creatinine ( $\mathrm{SCr}$ ) and blood urea nitrogen $(\mathrm{BUN})$ as well as creatinine clearance $(\mathrm{CrCl})$ were determined and recorded before the start of interventions, every other day during therapy, and 12 hours after the last dose of VCM in 10th day of treatment for all participants. Finally, the mean values of the measured parameters were compared between the groups.

Results: The mean values of SCr were significantly lower in drug group compared to placebo at the 4 th $(0.85 \pm 0.18$ vs. $0.98 \pm 0.22, P=0.016)$ and 6 th $(0.83 \pm 0.18$ vs. $0.95 \pm 0.21, P=0.029)$ days. Also, $\mathrm{CrCl}$ was significantly higher in the drug group at the 4 th day compared to placebo $(105.82 \pm 20.09$ vs. $94.67 \pm 20.53, P=0.041)$. Regarding VCM-induced AKI, no case was reported in any group.

Conclusion: Lycopene has the potential for diminution of VCM-induced nephrotoxicity (VIN). However, more investigations with larger sample size are necessary to confirm this effect.

Trial Registration: Present study was registered at the Iranian website (https://www.irct.ir/ trial/46859) for registration of clinical trials (identifier: IRCT20171030037093N37).
\end{abstract}

Implication for health policy/practice/research/medical education:

It has been proposed that oxidative stress plays a crucial role in vancomycin-induced nephrotoxicity (VIN). The present study aimed to investigate the nephroprotective effects of lycopene, as a powerful antioxidant, on VIN. Lycopene has the potential for reduction of VIN. However, more investigations with larger sample size are necessary to confirm this effect.

Please cite this paper as: Haghighipour S, Soltani R, Anjomshoa A. The protective effect of lycopene supplement against vancomycin-induced nephrotoxicity; a randomized double-blind placebo-controlled clinical trial. J Renal Inj Prev. 2020; 9(4): e32. doi: 10.34172/jrip.2020.32.

\section{Introduction}

Vancomycin (VCM) is a bactericidal antibiotic with a molecular weight of $1.446 \mathrm{kD}$ that stops the synthesis of cell wall in gram-positive bacteria (GPB) (1). This antibiotic is a keystone drug for the management of severe infectious conditions caused by GPBs. Prescription of VCM is effective for management of bacterial infections that triggered by Staphylococcus epidermidis, Streptococcus pyogenes, Streptococcus pneumoniae, Streptococcus viridans, Clostridium, Corynebacterium, Staphylococcus aureus, and certain bacillus species (2-4). However, it is not selected as a first-line treatment because of its reported side effects (5). Renal toxicity, ototoxicity, phlebitis, chills, exanthema and fever are some of the side effects of VCM $(4,6)$. Additionally, the dose, concentration of the infusion solution, and infusion rate are important factors for 
occurrence of the aforementioned toxicities.

The precise underlying mechanism of vancomycininduced nephrotoxicity (VIN) is not well known; however, it has been proposed that free radicals play a crucial role in the pathogenesis of VIN (7). It has been reported that urinary levels of malondialdehyde (MDA), as an important marker of lipid peroxidation, and $\mathrm{N}$-acetylD-glucosaminidase, as a marker of renal tubular injury, increase following VCM administration in laboratory animals and conversely, the activity of some antioxidant enzymes such as superoxide dismutase (SOD) and catalase (CAT) decreases in them (8-10). Due to the key role of oxidative stress in the pathogenesis of VIN, treatment with natural antioxidants is considered as a potntially useful therapeutic option.

Lycopene, a member of carotenoid family, is found plentifully in tomato and related products (11). Due to the presence of a large number of conjugated double bonds, lycopene is considered as an important natural antioxidant (12). Bae et al reported that intake of lycopene reinforces the antioxidant system and inhibits lipid peroxidation in cell membrane. They also revealed that lycopene blocks the chain of nuclear factor kappa B (NF$\mathrm{kB})$ activation and tumor necrosis factor-alpha (TNF- $\alpha$ ) generation (13).

\section{Objectives}

The present study aimed to investigate the possible nephroprotective effects of lycopene supplementation against VIN in humans.

\section{Patients and Methods \\ Study design}

The present double-blind randomized placebo-controlled clinical trial was performed from September 2019 to February 2020 in infectious diseases department of Alzahra hospital, Isfahan, Iran affiliated to Isfahan University of Medical Sciences (IUMS). Demographic data of patients admitted to the department who required treatment with VCM were documented in special forms. The inclusion criteria for the participation in our study were: (1) age between 18 and 60 years; (2) receiving $\mathrm{VCM}$ at a dose of $1 \mathrm{~g}$ every 12 hours for any indication; (3) creatinine clearance $(\mathrm{CrCl})$ more than $90 \mathrm{~mL} / \mathrm{min}$; (4) no positive history of acute kidney injury (AKI); (5) lack of underlying diseases causing renal impairment (e.g., diabetes mellitus and hypertension); (6) lack of any underlying kidney disorders such as glomerulonephritis, polycystic kidney disease, kidney stones, interstitial nephritis, and renal carcinoma; (7) no concurrent use of any other antioxidant supplements including vitamins $\mathrm{C}$ and $\mathrm{E}$; and (8) no concurrent use of other nephrotoxic drugs such as aminoglycosides, amphotericin B, piperacillin/tazobactam, cyclosporine, tacrolimus, and furosemide. Development of AKI due to any reason other than VCM and allergic reaction to lycopene were considered as the exclusion criteria (Figure 1).

Subjects who met the inclusion criteria were randomly divided into two groups of drug (lycopene) and placebo. Block randomization method was used for random allocation using blocks of 4 . Participants in the drug group

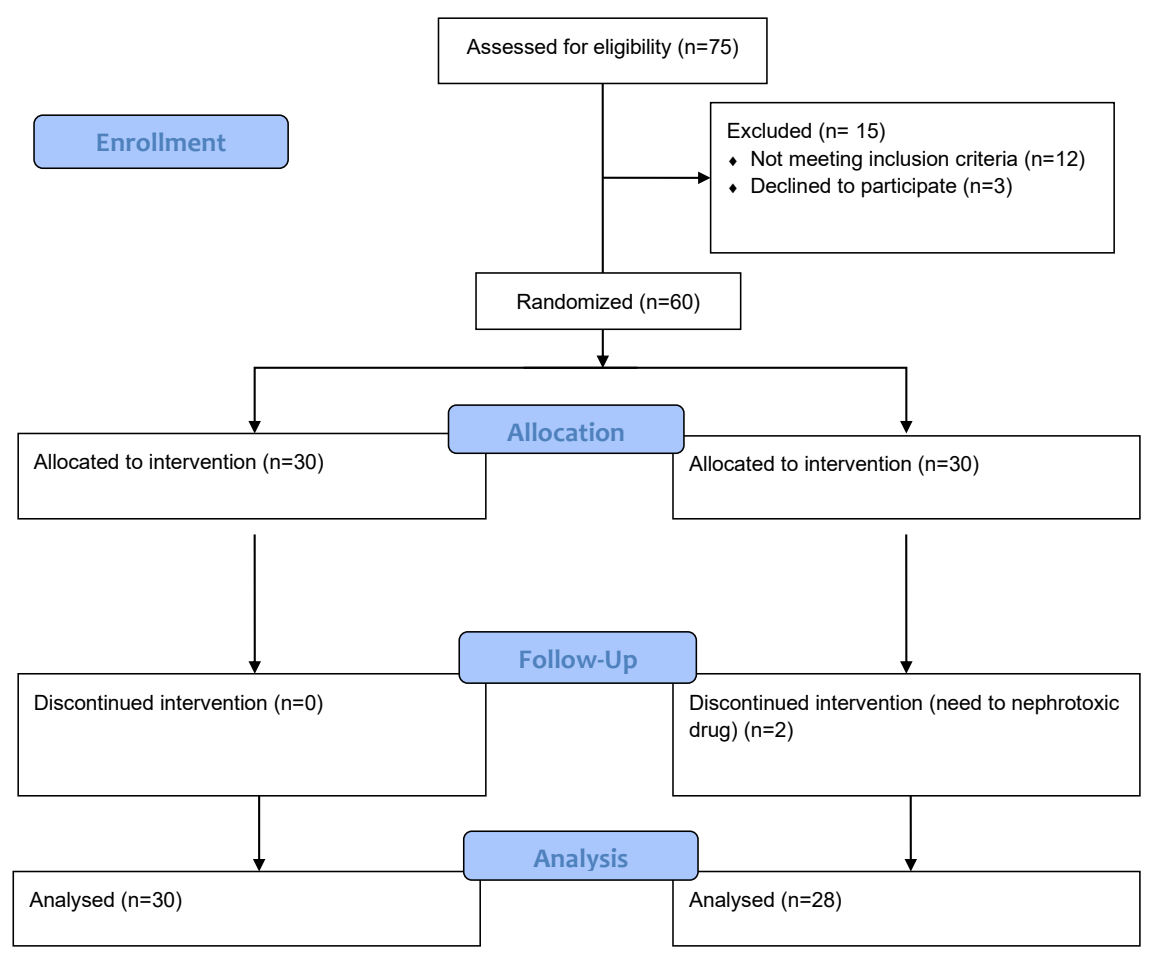

Figure 1. Flow chart of the study. 
received lycopene tablets $25 \mathrm{mg}\left(21^{\text {st }}\right.$ Century, U.S.A) daily for ten days started concurrently with VCM initiation. The subjects in the placebo group received placebo tablets (manufactured by the faculty of Pharmacy, IUMS, with the size, shape, and color similar to lycopene tablets) along with VCM. If VCM was discontinued earlier than ten days due to any reason except for AKI (e.g., thrombocytopenia and hypersensitivity reactions), the patient was excluded from the study.

\section{Outcome variables}

Outcome variables including serum levels of creatinine (SCr) and blood urea nitrogen (BUN) as well as $\mathrm{CrCl}$ were recorded before the start of intervention (day 0 ), every other day during the study (days 2, 4, 6, and 8), and 12 hours after the last dose of VCM (end of intervention; day 10) for all subjects. The estimated glomerular filtration rate (eGFR) as a magnitude of $\mathrm{CrCl}$ was calculated using Cockcroft-Gault equation (14). Also, the number of cases presented with AKI after VCM administration in two study groups was recorded. AKI was defined according to the KDIGO (Kidney Disease Improving Global Outcomes) definition, as an absolute increase in SCr of at least $0.3 \mathrm{mg} /$ $\mathrm{dL}$ within 48 hours or by a $50 \%$ increase in SCr within 7 days from baseline value (15).

\section{Ethical issues}

The research followed the tenets of the Declaration of Helsinki. The study protocol was registered in the Iranian Registry of Clinical Trials (identifier: IRCT20171030037093N37; https://www.irct.ir/trial/46859). Moreover, the study was approved by the ethics committee of the Isfahan University of Medical Sciences (IR.MUI. MED.REC.1398.104). Accordingly, informed consent was obtained from all patients. This study was also extracted from the residential thesis of Ali Anjomshoa at the department of infectious diseases of this university (Thesis No. 398057).

\section{Statistical analysis}

The extracted data were analyzed by statistical package for social science (SPSS) software, version 25.0 (SPSS Inc., Chicago, IL, USA). The qualitative variables were reported as percentage (\%) and quantitative variables as mean \pm $\mathrm{SD}$. Independent samples $t$ test and repeated-measures ANOVA, respectively, were used for comparison of the mean values in each evaluated time point and comparison of changing trend between the groups. A $P$ value $<0.05$ was considered as statistically significant difference.

\section{Results}

Over the study period, 112 subjects were evaluated regarding eligibility that 60 of whom met the inclusion criteria. Two patients were excluded from the drug group because of the need for another nephrotoxic drug (piperacillin/tazobactam for one and amphotericin B for the other). Therefore, 28 and 30 individuals completed the study in drug and placebo groups, respectively. Sixteen patients in drug group (57.1\%) and 23 patients in placebo group $(76.7 \%)$ were male $(P=0.113)$. The mean \pm SD of patients age in drug and placebo groups was $45 \pm 12.02$ and $46.5 \pm 11.98$ years, respectively $(P=0.630)$.

The results of three evaluated parameters of kidney function including $\mathrm{SCr}, \mathrm{BUN}$, and $\mathrm{CrCl}$ are summarized in Table 1. As shown, the difference of baseline values for all variables was insignificant between the groups. Furthermore, although SCr was lower in drug group compared to placebo at all time points, the difference was statistically significant only at the $4^{\text {th }}(P=0.016)$ and $6^{\text {th }}$ $(P=0.029)$ days of intervention. Also, the trend of changes in $\mathrm{SCr}$ was significantly different between the groups $(P=0.038)$.

No significant difference was found for BUN between the two groups in any evaluated time points. Furthermore, the changing trend of this parameter was not significant between the groups $(P=0.720)$.

Regarding $\mathrm{CrCl}$, although the mean values were higher in the drug group compared to placebo at all evaluated times, the difference was statistically significant only at the $4^{\text {th }}$ day of intervention $(P=0.041)$. Also, the trend of changes in $\mathrm{CrCl}$ was significantly different between the groups $(P=0.021)$. During the study, no case of AKI occurred in both groups.

\section{Discussion}

Our study showed the potential effect of lycopene for prevention of $\mathrm{SCr}$ rise and $\mathrm{CrCl}$ reduction by VCM. However, due to no occurrence of AKI in the placebo group that may be related to low dose of VCM or short duration of treatment, the judgment about this effect is difficult and needs more studies with larger sample size.

Several conditions have been recommended as the predisposing factors for VIN $(14,15)$. It has been stated that older subjects, those receiving a longer period of treatment, and those who are seriously ill are mainly at risk for renal toxicity induced by VCM (16-18). It has been reported that VIN occurs in $10 \%-20 \%$ and $30 \%-40 \%$ of cases receiving conventional and high doses of VCM, respectively (7).

At the best of our knowledge, this is the first study on the preventive effect of lycopene against VIN. However, several animal and clinical studies have been performed on other nephrotoxic drugs. Mahmoodnia et al reported that consumption of lycopene supplement is related to decrease of complications due to nephrotoxicity induced by cisplatin in subjects with malignancies (19). In another study, Dai et al investigated nephroprotective effect of lycopene on colistin-induced nephrotoxicity in the experimental model (20). They proposed that coadministration of lycopene with the dose of $20 \mathrm{mg} / \mathrm{kg} / \mathrm{d}$ 
Table 1. Comparison of renal function parameters at the evaluated times

\begin{tabular}{|c|c|c|c|c|c|}
\hline \multirow{2}{*}{ Variable } & \multirow{2}{*}{ Time (day) } & \multicolumn{2}{|c|}{ Group } & \multirow{2}{*}{$P$ value $^{a}$} & \multirow{2}{*}{$P$ value } \\
\hline & & Drug (Lycopene) ( $n=28)$ & Placebo $(n=30)$ & & \\
\hline \multirow{6}{*}{$\mathrm{SCr}(\mathrm{mg} / \mathrm{dL})$} & 0 & $0.94 \pm 0.16$ & $0.95 \pm 0.14$ & 0.866 & \multirow{6}{*}{0.038} \\
\hline & 2 & $0.89 \pm 0.15$ & $0.94 \pm 0.19$ & 0.280 & \\
\hline & 4 & $0.85 \pm 0.18$ & $0.98 \pm 0.22$ & 0.016 & \\
\hline & 6 & $0.83 \pm 0.18$ & $0.95 \pm 0.21$ & 0.029 & \\
\hline & 8 & $0.87 \pm 0.17$ & $0.93 \pm 0.19$ & 0.242 & \\
\hline & 10 & $0.88 \pm 0.14$ & $0.95 \pm 0.23$ & 0.173 & \\
\hline \multirow{6}{*}{ BUN (mg/dL) } & 0 & $12.93 \pm 5.22$ & $12.03 \pm 4.72$ & 0.490 & \multirow{6}{*}{0.720} \\
\hline & 2 & $12.18 \pm 3.61$ & $11.87 \pm 4.50$ & 0.770 & \\
\hline & 4 & $11.14 \pm 4.16$ & $11.50 \pm 4.84$ & 0.760 & \\
\hline & 6 & $11.18 \pm 4.58$ & $10.47 \pm 4.15$ & 0.534 & \\
\hline & 8 & $10.86 \pm 2.92$ & $10.43 \pm 3.55$ & 0.623 & \\
\hline & 10 & $11.96 \pm 3.08$ & $11.00 \pm 3.56$ & 0.276 & \\
\hline \multirow{6}{*}{$\mathrm{CrCl}(\mathrm{ml} / \mathrm{min})$} & 0 & $94.68 \pm 17.02$ & $95.63 \pm 16.39$ & 0.821 & \multirow{6}{*}{0.021} \\
\hline & 2 & $100.18 \pm 16.02$ & $98.30 \pm 19.66$ & 0.699 & \\
\hline & 4 & $105.82 \pm 20.09$ & $94.67 \pm 20.53$ & 0.041 & \\
\hline & 6 & $108.64 \pm 24.10$ & $97.77 \pm 22.01$ & 0.078 & \\
\hline & 8 & $102.57 \pm 18.19$ & $100 \pm 22.84$ & 0.633 & \\
\hline & 10 & $101.43 \pm 21.04$ & $98.37 \pm 23.75$ & 0.601 & \\
\hline
\end{tabular}

The values are mean $\pm S D$.

$\mathrm{sCr}$, serum creatinine; BUN, blood urea nitrogen; $\mathrm{CrCl}$, creatinine clearance

a Independent Samples t-test, between-group difference at each time point.

${ }^{\mathrm{b}}$ Repeated-measures ANOVA, time* group interaction (changing trend difference between the groups).

can protect against nephrotoxicity induced by colistin in animals via activation of the Nrf2/HO-1 pathway (20). Also, in the experimental study performed by Bayomy et al in 2017, the protective effects of lycopene and rosmarinic acid on gentamicin-induced renal oxidative stress were investigated. They reported that rosmarinic acid and lycopene alone or combined have useful effects against gentamicin-induced renal damage (21).

Since the most possible underlying mechanism for VIN is increased production of free radicals (22), the positive effects of lycopene observed in our and above-mentioned studies could be due to its strong antioxidant activity.

As observed in Table 1 and mentioned in the results section, the alterations in $\mathrm{SCr}$ and $\mathrm{CrCl}$ during the 10day intervention period revealed a significant difference between the two groups, so that SCr had decreasing trend and $\mathrm{CrCl}$ had increasing trend until the $6^{\text {th }}$ day of intervention versus relatively stable values for both parameters in the placebo group. This difference along with significantly lower SCr and higher $\mathrm{CrCl}$ at some time points in the drug group compared to the placebo group shows the potential ability of lycopene for improvement or preservation of renal function in the setting of VCM administration.

In the current study, we were confronted with some limitations including low sample size, short duration of the study, and lack of VCM dosing based on serum concentrations. Larger sample size could reveal the exact rate of VCM-induced AKI in our population and subsequently determine the role of lycopene in the prevention of this adverse effect. Future studies are recommended to investigate the cellular and molecular aspects of the effects of lycopene on nephrotoxicity. Moreover, similar studies that use more specific renal biomarkers (e.g., neutrophil gelatinase-associated lipocalin) are necessary to make a definite statement about the nephroprotective effects of lycopene as these biomarkers differentiate impairment of glomerular filtration from that of tubular damage $(25,26)$.

\section{Conclusion}

Lycopene has the potential for reduction of VIN. However, more investigations with larger sample size are needed to confirm this effect.

\section{Limitations of the study}

Low sample size, short duration of the study, and lack of VCM dosing based on serum concentrations are our limitations of the study.

\section{Author's contribution}

$\mathrm{SH}$ and $\mathrm{RS}$ were the principal investigators of the present trial study. AA, RS, and SH were included in designing the trial. AA participated in writing the initial draft of the article. AA, RS, and SH confirmed the accuracy of any part of the manuscript.

\section{Conflicts of interest}

The authors declare that there are no conflicts of interest. 
Ethical considerations

Ethical issues (including plagiarism, data fabrication, double publication) have been completely observed by the authors.

\section{Funding/Support}

This study was supported by IUMS Deputy Research (Grant No. 398057).

\section{References}

1. Gupta A, Biyani M, Khaira A. Vancomycin nephrotoxicity: myths and facts. Neth J Med. 2011;69:379-83.

2. Yang X-Y, Xu J-Y, Wei Q-X, Sun X, He Q-Y. Comparative proteomics of Streptococcus pneumoniae response to vancomycin treatment. OMICS. 2017;21(9):531-9. doi: 10.1089/omi.2017.0098.

3. Power EG, Abdulla YH, Talsania HG, Spice W, Aathithan $S$, French GL. van A genes in vancomycin-resistant clinical isolates of Oerskovia turbata and Arcanobacterium (Corynebacterium) haemolyticum. J Antimicrob Chemoth. 1995;36:595-606.

4. Bruniera FR, Ferreira FM, Savioli LR, Bacci MR, Feder D, Pereira EC, et al. Endothelial, renal and hepatic variables in wistar rats treated with Vancomycin. Anais da Academia Brasileira de Ciências. 2014;86(4):1963-72.

5. Filippone EJ, Kraft WK, Farber JL. The nephrotoxicity of vancomycin. Clin Pharmacol Ther. 2017;102(3):459-69.

6. MacDougall $\mathrm{C}$, Chambers $\mathrm{H}$. Protein synthesis inhibitors and miscellaneous antibacterial agents. In: Goodman and Gilman's the Pharmacological Basis of Therapeutics. New York: McGraw-Hill; 2011. p. 1521-47.

7. Nishino Y, Takemura S, Minamiyama Y, Hirohashi K, Ogino $\mathrm{T}$, Inoue $\mathrm{M}$, et al. Targeting superoxide dismutase to renal proximal tubule cells attenuates vancomycin-induced nephrotoxicity in rats. Free Radic Res. 2003;37:373-9.

8. Celik I, Cihangiroglu M, Ilhan N, Akpolat N, Akbulut HH. Protective effects of different antioxidants and amrinone on vancomycin-induced nephrotoxicity. Int J Basic Clin Pharmacol. 2005;97:325-32.

9. Cetin H, Olgar Ş, Oktem F, Ciris M, Uz E, Aslan Ç, et al. Novel evidence suggesting an anti-oxidant property for erythropoietin on vancomycin-induced nephrotoxicity in a rat model. Clin Exp Pharmacol. 2007;34:1181-5.

10. Hodoshima N, Nakano Y, Izumi M, Mitomi N, Nakamura $\mathrm{Y}$, Aoki $\mathrm{M}$, et al. Protective effect of inactive ingredients against nephrotoxicity of vancomycin hydrochloride in rats. Drug Metab Pharmacok. 2004;19:68-75.

11. Fraser GE, Jacobsen BK, Knutsen SF, Mashchak A, Lloren JI. Tomato consumption and intake of lycopene as predictors of the incidence of prostate cancer: the Adventist Health Study-2. Cancer Causes Control. 2020;31:341-351. doi: 10.1007/s10552-020-01279-z.
12. Fachinello MR, Gasparino E, Partyka AVS, de Souza Khatlab A, Castilha LD, Huepa LMD, et al. Dietary lycopene alters the expression of antioxidant enzymes and modulates the blood lipid profile of pigs. Anim Prod Sci. 2020;60:806-14.

13. Bae JW, Bae J-S. Barrier protective effects of lycopene in human endothelial cells. J Inflamm Res. 2011;60:751-8.

14. Mangiagalli MG, Martino PA, Smajlovic T, Guidobono Cavalchini L, Marelli SP. Effect of lycopene on semen quality, fertility and native immunity of broiler breeder. British poultry science. 2010;51:152-7.

15. Biddle MJ, Lennie TA, Bricker GV, Kopec RE, Schwartz SJ, Moser DK. Lycopene dietary intervention: a pilot study in patients with heart failure. J Cardiovasc Nurs. 2015;30:20512.

16. Wolak T, Sharoni Y, Levy J, Linnewiel-Hermoni K, Stepensky D, Paran E. Effect of tomato nutrient complex on blood pressure: a double blind, randomized dose-response study. Nutrients. 2019; 11(5): 950. doi: 10.3390/nu11050950

17. Prendecki M, Blacker E, Sadeghi-Alavijeh O, Edwards $\mathrm{R}$, Montgomery $\mathrm{H}$, Gillis S, et al. Improving outcomes in patients with acute kidney injury: the impact of hospital based automated AKI alerts. Postgrad Med J. 2016;92:9-13. doi: 10.1136/postgradmedj-2015-133496.

18. Bamgbola O. Review of vancomycin-induced renal toxicity: an update. Ther Adv Endocrinol. 2016;7:136-47.

19. Lodise TP, Lomaestro B, Graves J, Drusano G. Larger vancomycin doses (at least four grams per day) are associated with an increased incidence of nephrotoxicity. Antimicrob Agents Chemother. 2008;52:1330-6. doi: 10.1128/AAC.01602-07

20. Ingram PR, Lye DC, Tambyah PA, Goh WP, Tam VH, Fisher DA. Risk factors for nephrotoxicity associated with continuous vancomycin infusion in outpatient parenteral antibiotic therapy. J Antimicrob Chemother. 2008;62:16871. doi: $10.1093 / \mathrm{jac} / \mathrm{dkn} 080$.

21. Vandecasteele SJ, De Vriese AS. Recent changes in vancomycin use in renal failure. Kidney Int. 2010;77:760-4.

22. Mahmoodnia L, Mohammadi K, Masumi R. Ameliorative effect of lycopene effect on cisplatin-induced nephropathy in patient. J Nephropathol. 2017;6:144-9. doi: 10.15171/ jnp.2017.25

23. Dai C, Tang S, Deng S, Zhang S, Zhou Y, Velkov T, et al. Lycopene attenuates colistin-induced nephrotoxicity in mice via activation of the Nrf2/HO-1 pathway. Antimicrob Agents Chemother. 2015;59:579-85.

24. Bayomy NA, Elbakary RH, Ibrahim MA, Abdelaziz EZ. Effect of lycopene and rosmarinic acid on gentamicin induced renal cortical oxidative stress, apoptosis, and autophagy in adult male albino rat. Anat Rec. 2017;300(6):1137-49.

25. Zamoner W, Prado IRS, Balbi AL, Ponce D. Vancomycin dosing, monitoring and toxicity: Critical review of the clinical practice. Clin Exp Pharmacol Physiol. 2019;46:292301. doi: 10.1111/1440-1681.13066.

Copyright (c) 2020 The Author(s); Published by Nickan Research Institute. This is an open-access article distributed under the terms of the Creative Commons Attribution License (http://creativecommons.org/licenses/by/4.0), which permits unrestricted use, distribution, and reproduction in any medium, provided the original work is properly cited. 